\title{
Espacio Público y Políticas Sociales para la niñez y adolescencia
}

\author{
Laura Eugenia Garcés*
}

\section{Resumen}

Entre las múltiples transformaciones sociales de nuestro tiempo podemos reconocer las referidas al papel de la sociedad civil (a través de sus distintas organizaciones y movimientos) en los asuntos de interés público, a su intervención en la atención de determinadas necesidades sociales, como así también a su rol en la defensa y/o promoción de los derechos humanos y de las diversidades culturales, étnicas y de género. Dichas transformaciones no pueden considerarse de manera aislada sino que están vinculadas, por un lado, a la complejización y diversificación de las necesidades y demandas sociales y, por otro, a los cambios ocurridos previa y concomitantemente en el Estado. Justamente la discusión sobre el papel de la sociedad civil se desplegó en América Latina en un contexto de retraimiento, deserción y deterioro creciente de los Estados de Bienestar, como así también, en el debate políitico y políitico académico se produjo un desplazamiento hacia "la sociedad civil" como lugar de lo común y lo general frente a los Estados autoritarios de la región. Estas nuevas formas de vinculación suponen redefinir la noción de políticas públicas, examinar con detención las responsabilidades, alcances y límites del papel de cada uno de estos actores y de sus posibles articulaciones, lo que conllevaría, ciertamente la definición de una "nueva institucionalidad". En este trabajo, y como un ejemplo de lo referido, se analiza la participación de las organizaciones de la sociedad civil que se ocupan de niños y adolescentes en la provincia de San Juan con relación a políticas estatales en este área, particularmente en los programas sociales de promoción y asistencia.

\section{Palabras clave}

Políticas Sociales, Estado de Bienestar, Espacio Público, Niñez, Adolescencia, Sociedad Civil, América latina, ONGés,

\section{Abstract \\ PUBLIC SPACE AND SOCIAL POLITICAL FOR CHILDHOOD AND ADOLESCENCE}

Amid the multiple social transformations of our time, we can recognize those referred to the role of the civil society (through its different organizations and movements) in the subjects of public interest, its intervention in the consideration of certain social needs, as well as its roll in the defence and/or promotion of the human rights and the cultural, ethnic diversities and gender. These transformations cannot be considered in an isolated way but there is a link, on the one hand, to the intricacy and diversification of the social needs and demands and, on the other hand, to the changes happened previously and concomitantly in the State. Precisely, the discussion on the role of the civil society unfolded in Latin America in a context of retirement, desertion and increasing deterioration of the States of Welfare, as well as in the academic and political debate, there took place a displacement towards "the civil society" as a common and general place to the authoritarian States of the region. These new forms of entailment are supposed to redefine the notion of public policies, to firmly examine the responsibilities, scopes and limits of the role of each one of these actors and their possible connections, which would entail, undoubtedly, the definition of a "new institution's spirit". In this work, and, as an example of the issue referred to herein, the participation of the organizations of the civil society who take care of children and adolescents in the province of San Juan is analyzed in relation to state policies in this area, particularly in the social programs of support and assistance.

\section{Key words}

Social political, State of Welfare, Public Space, Childhood, Adolescence, Civil Society, Latin America, Ong's.

* Magíster, Facultad de Ciencias Sociales- Universidad Nacional de San Juan - Becaria doctoral CONICET- Doctoranda en Ciencias Sociales Facultad de Ciencias Sociales UBA, Argentina.

\section{Presentación}

$\mathrm{D}$ esde la sociedad civil, aparecen nuevos actores y movimientos sociales que buscan satisfacer intereses particulares, preservar sus identidades culturales, generar espacios propios de expresión social y defender el respeto por sus derechos como es el caso de las asociaciones de base, ONG's y movimientos sociales de defensa de los derechos del niño y del adolescente. Por su parte, el Estado tiene un papel protagónico en el reconocimiento jurídico de los derechos humanos en general, como también en el establecimiento de garantías y mecanismos institucionales para hacerlos efectivos, siendo, justamente, uno de sus roles más importantes: el ser garante de los derechos a quien los ciudadanos pueden exigir y demandar su cumplimiento. Podemos decir que, en este sentido, brinda respuestas de carácter más bien general que tienen que ver con arbitrar las condiciones necesarias para que esos derechos se hagan efectivos, función a la que no puede renunciar. 
Sin embargo, una de las limitaciones del accionar estatal es no brindar respuestas a demandas particulares vinculadas, por ejemplo, a la diversidad cultural, respuestas que pueden encontrarse en las diferentes organizaciones y movimientos sociales nucleados en torno a intereses especiales.

Estos cambios ocurridos en el Estado y en la sociedad civil implicarían, entre otras cosas, un mayor protagonismo y participación de las organizaciones sociales en los asuntos públicos. Sin embargo, esta afirmación muchas veces aparece en el discurso neoliberal como una "justificación” ante la ausencia o retirada del Estado de sus funciones esenciales respecto a las problemáticas sociales, ámbito en los que anteriormente ha tenido un rol protagónico. Se promueve la intervención de la sociedad civil, especialmente en la cuestión social negando, al mismo tiempo, al Estado como un actor protagónico tanto en el ámbito económico como en el ámbito social. “....La propuesta política del enfoque neo-conservador fundamenta la ampliación de la esfera de acción de la sociedad civil básicamente en la negación del Estado dentro de la institucionalidad deseable"'. Las críticas a la figura estatal llevaron entonces a trasladar muchas de las funciones y tareas asignadas previamente al Estado, a la sociedad civil ${ }^{2}$. Los argumentos esgrimidos respecto a este traslado están vinculados fundamentalmente al mayor conocimiento de la comunidad local respecto a los problemas sociales que los afectan y a su cercanía para solucionarlos; a la mayor eficiencia de las organizaciones de la sociedad civil en la prestación de servicios sociales; a las posibilidades de ejercer un mayor control de los recursos del Estado por parte de estas organizaciones y a la excesiva burocratización, ineficiencia y falta de transparencia en la provisión de servicios sociales por parte del Estado.

Gran parte de estos discursos están fundados en una visión dicotómica sobre el Estado y la sociedad

1 CUNILL GRAU, Nuria, 1995, p. 1.

2 Este planteo está presente también en JEREZ, Ariel, 1997 p. 42 y en DONATI, Pierpaolo, 1999, p. 113. civil, sobre lo público y lo privado, sin considerar el carácter difuso y móvil de los límites entre el Estado y la sociedad civil en cada momento histórico, así como la naturaleza compleja, ambigua, $\mathrm{y}$ a veces tensa, de las nuevas vinculaciones entre ambos actores.

Estas nuevas formas de vinculación implican, entre otras cosas, la redefinición del concepto de políticas públicas, entendiéndolas como una articulación de esfuerzos entre el Estado y la sociedad civil. Lo anterior deriva en un análisis de las responsabilidades, alcances y límites (aunque a veces difusos) del accionar de cada uno de estos actores y de sus posibles articulaciones. Podemos hablar, así, de una "nueva institucionalidad" en la que se redefinen tanto los roles del Estado y los de la sociedad civil como las relaciones y articulaciones entre ambos.

Partiendo de estos supuestos, en este trabajo, analizaré brevemente la participación de las organizaciones de la sociedad civil que se ocupan de niños y adolescentes en la provincia de San Juan con relación a políticas estatales en este área, particularmente en los programas sociales de promoción y asistencia.

\section{Acerca del concepto de sociedad civil}

El concepto de sociedad civil no es unívoco ya que, como plantea Barber ${ }^{3}$, podemos demarcar tres distintos puntos de vista sobre la sociedad civil: la perspectiva libertaria que la considera como sinónimo de sector privado; la perspectiva comunitaria que la considera como sinónimo de comunidad y la auténtica perspectiva democrática que la define como un tercer sector mediador entre el gobierno y el mercado, y rechaza la oposición diametral de los sectores públicos y privados postulando en su lugar un tercer sector mediador de compromiso social. La sociedad civil supone, además, ciudadanos actuando colectivamente en una esfera pública para

3 BARBER, Benjamín, 2000, p. 15-16 y 24-25. 
expresar sus intereses e ideas, alcanzar objetivos comunes y realizar demandas al Estado.

En el sentido de esta última perspectiva, Cohen y Arato, haciendo la salvedad del riesgo de tratar de definir un término usado en muchos contextos diferentes, con una historia conceptual larga y en evolución, nos brindan una definición operativa de sociedad civil entendiéndola como "una esfera de interacción social entre la economía y el Estado, compuesta ante todo de la esfera íntima (en especial la familia), la esfera de las asociaciones (en especial las voluntarias), los movimientos sociales y las formas de comunicación pública” ${ }^{4}$.

Sin embargo, si bien como esfera se distingue de la sociedad política y económica, éstas surgen de la sociedad civil y comparten con ésta algunas de sus formas de organización y comunicación. La diferencia está en que los actores de la sociedad política y económica participan directamente en el poder del Estado y en la producción económica respectivamente, a los cuales procuran controlar y manejar.

Estas tres esferas se interpenetran entre sí: el poder político de la sociedad civil no está relacionado directamente con el control o conquista del poder, pero sí con la generación de influencia mediante la actividad de sus asociaciones y la discusión no restringida en la esfera pública. Luego, esta influencia podrá ser canalizada por la sociedad política, fundamentalmente a través de los parlamentos, para lograr cambios en el Estado. Por su parte, en el ámbito de la economía, por ejemplo, la legalización de los sindicatos y las negociaciones colectivas, son muestras de la influencia de la sociedad civil sobre la esfera económica.

Hablar de “interpenetración” entre las distintas esferas de la vida social implica, entre otras cosas, distanciarnos de las visiones dicotómicas sobre el Estado y la sociedad civil, sobre lo público y lo privado, considerando, como ya señalamos, el

4 COHEN, Jean y ARATO, Andrew, 2000, p. 8. carácter difuso y móvil de los límites entre ambas esferas en cada momento histórico, así como la naturaleza compleja, ambigua y a veces tensa de sus formas de relacionamiento.

\section{Acerca del concepto de Política Social}

Siguiendo los planteos anteriores y a base de los desarrollos teóricos de Oszlak y O’Donell ${ }^{5}$ sobre las políticas públicas, consideramos a las políticas sociales a partir de las mutuas interpenetraciones entre el Estado y la sociedad civil; es decir a partir de relaciones bidireccionales de poder, influencia, negociación, cooptación ente los actores que intervienen en las políticas, sean estos pertenecientes a las diferentes áreas estatales como a distintos tipos de organizaciones y movimientos de la sociedad civil.

La política estatal es definida por estos autores como "el conjunto de acciones y omisiones que manifiestan una determinada modalidad de intervención del Estado en relación con una cuestión que concita la atención, interés, movilización de otros actores de la sociedad civil...” . Las cuestiones involucran a actores ${ }^{6}$ que pueden hallarse objetiva y/o subjetivamente afectados por las mismas y que toman también posición frente a estas cuestiones que los afectan, adoptando políticas cuyas consecuencias pueden influir considerablemente en el proceso de resolución de las mismas. Por lo tanto, la política social no puede ser explicada y entendida con prescindencia de las políticas de otros actores y de las mutuas vinculaciones entre las distintas unidades estatales y entre éstas y las diferentes organizaciones de la sociedad civil.

Ahora bien, como referíamos en la presentación, esta característica de la política social como "ar-

5 OSZLAK, Oscar y O’DONNELL, Guillermo, 1982.

6 Estos actores pueden ser clases, organizaciones, grupos, e incluso, individuos ubicados estratégicamente en el sistema de poder. 
ticulación entre el Estado y la Sociedad Civil” aparece en el discurso y en la política neoliberal de reforma del Estado y es resaltada como uno de los ejes más importantes en las definiciones de políticas sociales y en los diseños de programas sociales, fundamentalmente a partir de los '90. Ello implica que esta definición no pueda entenderse sin considerar previamente los cambios ocurridos en el Estado en esta década, especialmente los referidos al régimen de Estado de Bienestar de nuestro país, ya que el desarrollo de la sociedad civil como 'tercer sector' orientado a la prestación de servicios sociales se vincula a los componentes liberal-residual del régimen de bienestar social.

Dada la extensión y objetivo del presente trabajo, no nos ocuparemos de describir en profundidad este último punto teniendo en cuenta, además, que las transformaciones del Estado en materia económica y social han sido ampliamente desarrolladas en el debate de las Ciencias Sociales ${ }^{7}$. Nos centraremos, entonces, en las formas de vinculación entre organizaciones de la sociedad civil y un ámbito estatal, el de la Política Social, intentando responder la siguiente interrogante: ¿Cómo se invoca a la sociedad civil?, ¿en qué contextos?, ¿de qué tipo de organizaciones de la sociedad civil se trata? ¿cuáles son los límites y posibilidades de estas organizaciones en relación a las políticas sociales?

Articulación Estado-Sociedad Civil en los programas sociales destinados a la niñez y adolescencia en la provincia de San Juan

Una primera aclaración sobre este apartado es que, de ese binomio relacional tan amplio "Estado-sociedad civil”, analizaremos concretamente la articulación entre los programas sociales de

7 Rubén Lo Vuolo, Alberto Barbeito, Aldo Isuani, Emilio Tenti, Estela Grassi, Claudia Danani, entre otros autores argentinos, han realizado numerosos aportes en este tema, fundamentalmente con relación al nuevo papel del Estado en materia social (focalización, descentralización, privatización, financiamiento de la política social, entre otros). promoción y de asistencia destinados a la niñez y adolescencia dependientes de la Dirección de Niñez, Adolescencia y Familia de la Provincia de San Juan (con relación al ámbito estatal) y las organizaciones sociales que participan de estos programas (con relación a la sociedad civil).

La mencionada Dirección lleva adelante el Plan de Protección Integral de Niños y Adolescentes, sustentado en el marco legal de la Convención Internacional de los Derechos del Niño y, entre los objetivos de ese plan, vinculados al papel de la sociedad civil, encontramos que se propone la creación de un Consejo Consultivo Provincial de Protección a la Niñez y Adolescencia con la participación de las autoridades provinciales y municipales, otras áreas del Estado y Organizaciones de la Sociedad Civil (OSC) abocadas a los temas de la infancia. Además, tiene como objetivo "promocionar la participación de las OSC en temas relacionados con la infancia y adolescencia incentivar y apoyar aquellas con largas trayectorias de trabajo en la provincia".

Para hacer efectivos estos lineamientos se promueve la descentralización, fortaleciendo las áreas de niñez y familia a nivel municipal, se crean redes sociales y se focaliza en la gestión asociada con Municipios, OSC y sectores privados de producción que apoyan estas instancias. La Dirección define una "Política de Fortalecimiento de la Sociedad Civil” fundamentando tal definición en "la imposibilidad que tiene el Estado para dar respuestas a todas las demandas sociales cada vez más complejas y numerosas que superaron la capacidad operativa del mismo. Las políticas públicas comenzaron a incorporar, a partir de la década del '90, el ideario de un trabajo conjunto con instituciones de la sociedad, desde el aspecto metodológico, se replantean los estilos de Planificación Estratégica y Gestión Asociada $\{. . .$.$\} Comienza a instalarse la$ necesidad de descentralizar acciones y asumir un

8 Fuente: "Política de Infancia. Monitoreo y evaluación del Plan de Protección Integral a la Niñez y Adolescencia", bienio 2004-2005 Dirección de la Niñez, Adolescencia y Familia de la Provincia de San Juan. 
rol de nexo con el sector civil, la crisis de fines de los '90 e inicio del milenio da un protagonismo a la sociedad civil, al ciudadano, quien exige espacios de participación y quiere intervenir en las decisiones que lo afectan”.

Por su parte, analizando el tipo de organizaciones de la sociedad civil que participan en los programas de esta Dirección, se trata, en su mayoría, de 'asociaciones de base territoriales' y, en menor medida, de 'organizaciones no gubernamentales' (ONG’s) dedicadas a la niñez y adolescencia la provincia de San Juan. Siguiendo la tipología de “Organizaciones sin fines de lucro” elaborada por Campetella, González Bombal y Roitter ${ }^{9}$ podríamos ubicar a estas organizaciones dentro del grupo de asociaciones civiles pero con características particulares en cada caso.

Las $O N G$ 's son a organizaciones ligadas a la promoción del desarrollo social o la defensa de derechos que generalmente cuentan con apoyo de la cooperación internacional. Actúan prestando o intermediando servicios, o bien haciendo "advocacy" en temas como ambientalismo, derechos humanos, en general reivindicando derechos colectivos, ya sea frente al Estado o frente a empresas. Aunque legalmente aparecen registradas como asociaciones civiles, se distinguen como universo en tanto ejercen presión y presencia en el espacio público. Mientras, las organizaciones de base aluden a asociaciones de los sectores populares, que surgen ligadas al lugar geográfico de residencia de sus integrantes, usualmente villas o asentamientos. Normalmente permanecen como organizaciones más informales, sin adquirir estatuto legal, debido a los costos que ello supone. Suelen contar con la asistencia técnica de organizaciones no gubernamentales y, generalmente, tienen objetivos concretos en relación a la demanda de mejoras de su situación o llevar a cabo actividades más permanentes como apoyo escolar, guarderías, comedores infantiles, ollas populares, entre otras. ${ }^{10}$

9 CAMPETELLA, Andrea; GONZALEZ BOMBAL, Inés y ROITTER, Mario, 2000, p. 5-8-

10 Estas características se vinculan con lo que JEREZ Y REVILLA (1997, p. 30) denominan 'Asociacionismo civil'
En el caso que analizamos, las ONG's que han persistido en el tiempo en su vinculación formal con el Estado a través de distintas políticas sociales, son aquellas que provienen de la Iglesia Católica y podemos señalar sus orígenes con el advenimiento de la democracia, con un objetivo pastoral que con el tiempo fue derivando en distintas acciones sociales y adquiriendo un matiz vinculado a la promoción de derechos.

El resto de las organizaciones con las que se vincula la Dirección en su mayoría pueden caracterizarse como "de base", vinculadas a lo comunitario y su surgimiento se ubica en los '90 y está vinculado a la crisis social característica de esa época concomitante a la retirada del Estado que describíamos anteriormente. En general, estas organizaciones surgen para atender a las comunidades más necesitadas que no encuentran respuestas en las instituciones estatales que tradicionalmente se ocupaban de la cuestión social, o bien, que dichas respuestas resultan insuficientes o retrasadas en el tiempo. Entre sus objetivos figuran por ejemplo: "satisfacer a corto plazo la necesidad de alimentación”; "brindar apoyo escolar”, “otorgar la copa de leche”, “contribuir al mejoramiento de las condiciones de vida de la población infantil más vulnerable”"11.

Su acción está orientada fundamentalmente a la atención de necesidades de las comunidades en las que se insertan e, incluso, de sus miembros, y no tiene en general un contenido ideológico ni direccionalidad política como acción colectiva extensible al conjunto de la sociedad. Podemos caracterizarlas como "...una de las modalidades básicas de afirmación y construcción de las nuevas identidades: abiertas, frágiles y precarias, orientadas a la acción local a partir de intereses puntuales y efímeros, dirigidas a resolver problemas, a reactivar la solidaridad y a promover la sociabilidad desde la inmediatez y singularidad de sus rasgos identitarios y desde la problemática surgida de los contextos de

11 Fuente: Registro único de Culto y ONG's. Dirección de Culto y ONG's. Ministerio de Gobierno de la Provincia de San Juan.

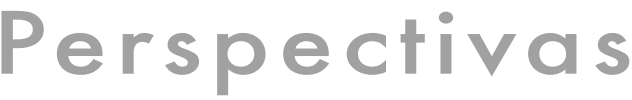


la vida cotidiana...”12. Respecto a su vinculación con el Estado, en primer lugar no puede obviarse que tienen una fuerte dependencia financiera respecto de éste, que en muchos casos condiciona hasta su sobrevivencia. Este financiamiento proviene de los distintas políticas y programas sociales en los que participan como organización. Esta participación, en la mayoría de los casos, no está vinculada a la expresión de intereses sociales organizados en el proceso de formulación de políticas y decisiones públicas, sino, fundamentalmente, al establecimiento de acciones solidarias para atender necesidades insatisfechas y a la ejecución de políticas y programas sociales nacionales o provinciales, como consecuencia del traspaso de responsabilidades del Estado hacia la sociedad, vinculado al proceso de descentralización.

Si bien uno de los objetivos que persigue la descentralización a través de la participación ciudadana es el aumento de la eficiencia y eficacia de estos proyectos y programas sociales, uno de los problemas más importantes es que, en el caso de las regiones o zonas más pobres y marginadas, la población no cuenta con los recursos necesarios para participar. Nos referimos, fundamentalmente, a recursos de orden simbólico vinculados a la formación educativa de estos grupos y a su capacidad organizativa para participar en la formulación y ejecución de los programas. Esta situación se pone en evidencia, por ejemplo, cuando tienen que elaborar un proyecto social para acceder a los recursos de determinado programa social ${ }^{13}$. Se "delegan" en la comunidad responsabilidades que en muchas ocasiones los transforman en "responsables" de las soluciones a sus problemas, cuando justamente se trata de comunidades vulnerables que necesitan

12 REIGADAS, M. Cristina, 2003, p. 294.

13 Algo similar ocurre "cuando se convoca a los sujetos sociales para que ejerzan un rol gerencial que invoca a los "saberes técnicos", son muy bajas las probabilidades de que opere una redistribución del poder a su favor: sólo quienes disponen de ese tipo de saber resultan privilegiados.... De allí se deriva que la participación social no puede basarse en el aporte de conocimientos especializados de parte de la sociedad." (CUNILL GRAU, 2004 , p. 81 una fuerte presencia del Estado para mejorar su nivel de vida.

Se da, así, una participación de la población muy acotada en la etapa final de ejecución de la política social y no en el proceso de su formulación o definición política donde se discutirían fines, contenidos y valores. Simplemente la discusión (cuando existe) se circunscribe al punto de vista técnico-instrumental y esto quizás se debe a que el impulso desde el Estado y desde los organismos donantes a la participación de la sociedad civil tenga como prioridad una cuestión técnico-administrativa: "como hacer que los escasos recursos destinados a la política social lleguen a aquellos que están en peor situación socio-económica para elevar el impacto per-cápita”. En definitiva, se trata de una cuestión en la que básicamente intervienen variables económicas de gasto-impacto (crisis fiscal mediante).

Desde esta perspectiva, el diseño de la política pública es una materia técnica y no política. Entenderla como cuestión política es poner de relieve que no hay “una” solución a los problemas políticos o sociales y enfatizar la importancia del proceso de deliberación y de dar "buenas razones" (deliberación discursiva entre los sujetos concernidos) ${ }^{14}$.

En este sentido, rescatamos la definición de la política como "una práctica social por la cual las modernas sociedades democráticas debaten abiertamente acerca del orden general, de las instituciones y de las necesidades del ámbito de la vida. Se trata de una práctica social pública de producción de significados (...) Lo público, en su conjunto, tiene que ver con la producción de recursos argumentales, con la constitución de sujetos con capacidad de hacer valer sus puntos de vista, necesidades e intereses (...) el sistema político es parte de 'lo político' pero no todo lo político”. ${ }^{15}$

Sin embargo, no podemos dejar de tener en cuenta que, para posibilitar ese proceso de deliberación

14 CUNILL GRAU, 1997, p. 168. 15 GRASSI, 2001, p. 103-104. 
discursiva son necesarios recursos de orden simbólico, acceso a la información necesaria para tomar posición, tanto como capacidades organizativas, especialmente cuando se trata de comunidades vulnerables. Esto no quiere decir que en estos ámbitos la deliberación no sea posible ya que justamente ella no está sostenida en saberes técnicos sino en valores y fines a partir de la consideración de las diferencias culturales. En este sentido, en la teoría Habermasiana de la ética del discurso "el principio de universalidad es un principio regulador del proceso discursivo por medio del cual los participantes razonan juntos sobre cuáles valores, principios e interpretaciones de necesidades merecen ser institucionalizadas como normas comunes” ${ }^{16}$.

En este escenario, de un desarrollo incipiente y débil de las organizaciones de la sociedad civil, se hace indispensable una presencia más protagónica del Estado, fundamentalmente respecto a dos de sus funciones indelegables: establecimiento de políticas de redistribución del ingreso y reconocimiento y establecimiento de mecanismos para hacer efectivos los derechos sociales. El Estado no puede abdicar sobre las responsabilidades públicas que le caben en el bienestar general de la población y, dado que su atención se basa en la idea de derechos y no de caridad, se convierte, en estos casos, en la posibilidad más importante para la apelación de los mismos. Esta responsabilidad se torna mucho más relevante en este tipo de comunidades desde donde surgen las organizaciones mencionadas, lo que no implica suponer ni promover la pasividad de dichas organizaciones y de los sujetos beneficiarios sino proveer las condiciones y recursos necesarios para posibilitar una participación responsable y democrática.

\section{Conclusiones}

A partir de lo expuesto en los apartados anteriores podemos caracterizar, en este caso, a la "articula-

16 COHEN Y ARATO, 2000, p.40. ción entre el Estado y la sociedad civil” como una “articulación funcional” en tanto deriva de la ejecución de una política o programa social, estrechamente vinculada al proceso de descentralización generado por el Estado en los últimos años.

La articulación tiene una fuerte preponderancia de la acción e iniciativa estatal y, al ser generada por el Estado, éste se constituye en el principal sostén de esta relación, además de la fuerte dependencia financiera que muchas de estas organizaciones tienen respecto al Estado.

El énfasis está puesto en le ejecución de las políticas sociales y no en la participación de las OSC en la planificación global de las mismas, así, más que de articulación podríamos hablar de un modelo de colaboración enmarcado en una perspectiva económico-administrativa cuyo eje central es la aplicación eficiente del gasto social.

Resulta necesario, entonces, repensar la articulación en el marco de un proyecto político conjunto entre ambas esferas; es decir, una articulación "más política que técnico-gerencial” donde se discutan fines, orientaciones, modelos y valores respecto a las definiciones de políticas y programas sociales. Estas discusiones podrían acercar a la definición conjunta de bien común que desean construir con su acción. Como plantea Nora Rabotnikof: “el 'espacio público ciudadano’ aparece como el lugar de recomposición de lo común y general, como el lugar de la integración social a través de la civilidad. La integración parece entenderse como referencia a lo general, como apelación a un conjunto de valores y procedimientos que orientan la conducta de los actores, y como procesamiento de consensos básicos y opiniones colectivas legitimadas por la participación y la discusión”. ${ }^{17}$

Será necesario, entonces, el establecimiento de canales, mecanismos e instrumentos que posibiliten la participación de la sociedad civil y la expresión de sus intereses en el proceso de formulación de

17 RABOTNIKOF, Nora, 1999, p. 201. 
políticas y decisiones públicas para que dicha participación no sea meramente "instrumental", es decir, la construcción de un espacio público como lugar de expresión de la sociedad civil.

Como planteáramos anteriormente, las principales dificultades para construir ese espacio, especialmente cuando existe un desarrollo incipiente y débil de las organizaciones de la sociedad civil, estarían vinculadas a las desigualdades sociales entre los actores que participan del mismo. Estas desigualdades no pueden ser "puestas en paréntesis" puesto que hacen que los que actores no puedan deliberar como iguales. Los sectores más vulnerables verán limitada su participación dada la falta de los recursos necesarios para hacerlo, fundamentalmente los de orden simbólico, de poder, de información y de organización a la hora de la discusión y deliberación para que sus necesidades, expectativas, ideas y valores lleguen a plasmarse en decisiones políticas. En este sentido, la función del Estado de integración social y de garante del bienestar general de la población es indelegable y la reducción de las desigualdades sociales estructurales se convierte en una de las condiciones necesarias para posibilitar la existencia de un espacio público en el sentido antes mencionado.

\section{BIBLIOGRAFÍA}

- $\quad$ BARBER, Benjamín (2000); Un lugar para, Paidós, Barcelona.

- $\quad$ CAMPETELLA, Andrea; GONZALEZ BOMBAL, Inés y ROITTER, Mario (2000); "Definiendo el sector sin fines de lucro en Argentina”, CEDES y John Hopkins University, Buenos Aires.

- $\quad$ COHEN, Jean y ARATO, Andrew (2000); Sociedad civil y teoría política, Fondo de Cultura Económica, México, 1992.

- CUNILL GRAU, Nuria (1995) "La rearticulación de las relaciones Estado-sociedad: en búsqueda de nuevos sentidos", en Revista del CLAD Reforma y Democracia, No. 4, Caracas.

- _ (1997), "La institucionalidad de la representación social: algunas premisas para su constitución”, en Repensando lo público a través de la sociedad: nuevas formas de gestión pública y representación social, Caracas, CLAD; Editorial Nueva Sociedad.

- _ _ (2004) "La descentralización de la descentralización de la política social ¿Qué hemos aprendido?”, en Descentralización y políticas sociales en América Latina, Gomá y Jourdana (eds.), Fundación CIDOB, Barcelona.

- $\quad$ DIRECCIÓN DE LA NIÑEZ, ADOLESCENCIA Y FAMILIA de la Provincia de San Juan: "Política de Infancia. Monitoreo y evaluación del Plan de Protección Integral a la Niñez y Adolescencia”, bienio 2004-2005.

- DONATI, Pierpaolo (1999): La ciudadanía societaria, Universidad de Granada.

- $\quad$ FRASER, Nancy (1993), "Repensar el ámbito público: una contribución a la crítica de la democracia realmente existente" en Revista Debate Feminista, año 4, Vol. 7, México.

- $\quad$ GARCIA DELGADO, Daniel; DE PIERO, Sergio (2002): "Articulación y relación Estado-Organizaciones de la Sociedad
Civil. Modelos y prácticas en la Argentina de las reformas de segunda generación”, FLACSO Argentina, Area Estado y Políticas Públicas.

- $\quad$ GRASSI, Estela (2001) “El descrédito de la política. Táctica y estrategias neoliberales”. En revista Encrucijadas N ${ }^{\circ}$ 6, UBA, Buenos Aires

- HABERMAS, J. (1990), "Soberanía popular como procedimento: um conceito normativo de espacio público”, en Novos Estudos CEBRAP, Sao Paulo No 26 .

- JELIN, Elizabeth (1996) “_Ciudadanía emergente o exclusión? Movimientos sociales y ONG en América latina en los años 90” en Revista Sociedad N ${ }^{\circ} 8$ Facultad de Ciencias Sociales, UBA.

- JEREZ, Ariel (coord.): ¿Trabajo voluntario o participación? Elementos para una sociología del Tercer Sector, Tecnos, Madrid, 1997.

- $\quad$ RABOTNIKOF, Nora (1993), "Lo público y sus problemas: notas para una reconsideración” en Revista Internacional de Filosofía Política, México-Madrid.

- $\quad$ OSZLAK, Oscar y O’DONNELL, Guillermo (1982): “Estado y políticas estatales en América Latina: hacia una estrategia de investigación”, en Revista Venezolana de Desarrollo Administrativo, $\mathrm{N}^{\circ} 1$, Caracas.

- $\quad$ RABOTNIKOF, Nora (1999); "Hegelianos, a sabiendas” en Lechner, Norbert y otros; Reforma del Estado y coordinación social, Plaza y Valdés Editores - Instituto de Investigaciones Sociales de la UNAM, México, D.F.

- $\quad$ REIGADAS, M. Cristina (2003), "Multiculturalismo: crítica a eurocentrismo o etapa superior del imperialismo" en REIGADAS, M. C. y CULLEN, C. (comp.), Globalización y nuevas ciudadanías, Ediciones Suárez, Mar del Plata. 